Canadian Oncology

Nursing Journal

Revue canadienne

de soins infirmiers

en oncologie

Volume 28, Issue 4 • Fall 2018

elSSN: 2368-8076 


\title{
Impact of a breast cancer care RN-coordinator program on patients' distress level
}

\author{
by Savitri Singh-Carlson, Frances Wong, Kris Trevillion, and Grace Reynolds
}

\section{ABSTRACT}

The purpose of this study was to determine whether patients receiving navigation supportive care from a Breast Cancer Care Coordinator $(B C C C)$, prior to initial oncology consultation at a British Columbia Cancer Agency, Abbotsford Centre (BCCA-AC), demonstrated different levels of anxiety and depression from those not receiving such support at the same BCCA centre. A retrospective review of the Psychological Screen for Cancer (PSSCAN) scores of new breast cancer patients seen for oncology consultation for the control cohort (receiving usual care) were compared to PSSCAN scores of those who had received care from a BCCC prior to the oncology consultation (the study cohort). A total of 91 PSSCANs were reviewed in the study, with 54 belonging to the treatment group and 37 to the control group. PSSCAN scores for anxiety and depression did not show significant differences between the two groups.

\section{INTRODUCTION}

$\mathbf{R}$ eceiving a new breast cancer diagnosis can invoke many emotional responses for women of all ages and ethnicity (Hewitt, Greenfield, Stovall, 2005; Pederson, Sawatzky, \& Hack, 2010). Reportedly, a program of routine screening for psychosocial distress may help to reduce increased levels of anxiety, as women go through the diagnostic process (Carlson, Groff, Maciejewski, \& Bultz, 2010). The American College of Surgeons has developed a Commission on Cancer standard that requires cancer programs to create a psychological distress screening tool, which addresses patients' emotional issues related to initial diagnoses and continued treatment (Buxton, Lazenby, Daugherty, et.al, 2014). Increased levels of anxiety and depression are amongst the feelings that most women experience and can inhibit one's ability to cope with breast

\section{ABOUT THE AUTHORS}

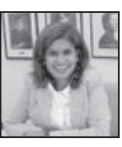

Address for correspondence: Savitri Singh-Carlson, PhD,

APHN-BC, FAAN, Professor, San Diego State University, School of Nursing, San Diego, CA, USA, 92182

Email: ssinghcarlson@sdsu.edu

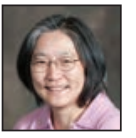

Frances Wong, RN, Breast Cancer Co-ordinator, BCCA, FVC

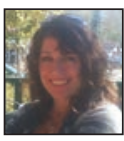

Kris Trevillion, MD, FRCPC(C), BCCA, FVC

Grace Reynolds, Professor, California State University, Long Beach, Health Care Administration, Graduate Program Director, Director, Center for Behavioral Research and Services

DOI:10.5737/23688076284250255 cancer diagnosis and treatment/s (Linden, Yi, Barroetavena, MacKenzie \& Doll, 2005; Campbell, Craig, Eggert, \& BaileyDorton, 2010; Pederson, Sawatsky, \& Hack, 2010; Case, 2011; Pederson \& Hack, 2011). Evidence indicates that specialized nurse navigators can help to decrease patients' stress and anxiety by providing psychosocial support and timely, appropriate information, and by ensuring continuity of care is structured (Campbell, Craig, Eggert, \& Bailey-Dorton, 2010; Case, 2011; Liebert, Parle, White, \& Rodger, 2011; Lim, Devi, \& Ang, 2011; Pederson \& Hack, 2011; Fillion, Cook, Veillette, Aubin, DeSerres et al.; 2012). This is confirmed by the National Coalition of Oncology Nurse Navigators and supported by studies with similar results (Winstead, 2012; National Cancer Institute, 2017).

Patients going through breast cancer diagnosis and treatment for the first time have expressed their value of a healthcare team approach for their cancer care, but have felt the lack of a structured healthcare system with coordination for all aspects of their cancer care (Hryniuk, Simpson, McGowan, \& Carter, 2014; Singh-Carlson, Gotz, 2014). Women have generally felt unsupported through the worst moments in their care experiences with breast cancer under the current arrangements, which are unstructured and uncoordinated (Drageset, Lindstrom, \& Underlid, 2009).

The British Columbia Cancer Agency, Abbotsford Centre (BCCA-AC) had identified the need for an oncology nurse in a specialized supportive role who cared for breast cancer patients prior to referral to the cancer centre. A specialized program of supportive care was implemented utilizing the role of breast cancer care coordinator (BCCC) and consisting of nurses with an oncology background, working both in the cancer centre and the associated hospital (Abbotsford Regional Hospital) breast health clinic (BHC). The BCCC's mission is to act as a link, a guide, and an information source that is supportive of the patient. Hryniuk, Simpson, McGowan, and Carter (2014) reported that supportive care is an emerging approach in the literature of cancer control and is based on the premise that people may generally benefit from having detailed health information, knowing the course of their treatment protocol, and playing a role in their own care-all in a team approach with their providers.

\section{Purpose}

The purpose of this study was to determine whether patients receiving navigation supportive care from a BCCC, prior to initial oncology consultation at the BCCA-AC centre, demonstrated different levels of anxiety and depression from those not receiving such support with usual care. This study used a retrospective chart review to compare patient responses for social support and anxiety/depression items of the Psychological Screen for Cancer (PSSCAN) questionnaire. 
PSSCAN is a cancer-specific screening instrument, developed for screening anxiety and depression for patients in the clinical setting (Linden, Yi, Barroetavena, MacKenzie \& Doll, 2005). This questionnaire is routinely used in the BCCA clinical setting as a screening tool for distress and was revalidated in this study (Linden, Vodermaier, MacKenzie, et.al., 2009).

\section{BREAST HEALTH CLINIC AND BREAST CANCER CARE CO-ORDINATOR ROLE}

The breast health clinic (BHC) was jointly developed by Abbotsford Regional Health and BCCA-AC. This unique collaboration between a hospital setting and a BCCA centre began in June 2011, as a result of a gap that was identified as lack of critical features, of coordinated communication and continuity for patients receiving an abnormal mammogram result (Trevillion, Singh-Carlson, Wong, \& Sherriff, 2015). This unique collaborative healthcare partnership, created a specialized program with an opportunity to provide focused care for women with suspicion of breast cancer, through the screening process to an actual diagnosis. The aim of the innovative BCCC program was to provide support to breast cancer patients from the start of the cancer care trajectory. The diagnostic process generally begins after a mammographic abnormality is detected following a visit to the BCCA's Screening Mammography Program (SMP). Patients are then referred to BHC with an appointment for further investigations and biopsy. During the BHC appointment, patients see a general practitioner or surgeon for a clinical exam and for mammogram +/- ultrasound, if necessary. Patients with biopsy positive breast cancer receive the pathology result from the surgeon at BHC soon after the results are known. They are generally seen by the BCCC the same day, but may be contacted by phone later that day or the following day to address any concerns. They will then be referred to an oncology consultation at the cancer centre. This process, from hearing the diagnosis to the first oncology consultation, may take an average of seven to 10 weeks.

The Breast Cancer Care Co-ordinator (BCCC) serves as an important link to the interdisciplinary team and is knowledgeable about the standards, protocols, policies, and best-practice guidelines at BCCA; the care pathway a patient might follow; the pathophysiology of breast cancer; related diagnostic procedures; breast cancer surgery/breast reconstruction surgery; and information on both systemic and radiation therapies. The patient who is newly diagnosed with breast cancer is offered a breast cancer information kit that contains the Intelligent Patient Guide to Breast Cancer book (BC Cancer Agency, 2017), as well as pamphlets on nutrition, post-surgery exercises, family support and survivorship information. The BCCC also helps in supporting emotional needs and resources through the initial nursing assessment process. The BCCC provides patients with information on available community resources and refers them to the Patient and Family Counseling Service at BCCA. This form of referral from all healthcare workers for psychosocial services is reported as the best predictor of decreased anxiety and depression in full screening and triage conditions (Carlson, Groff, Maciejewski, \& Bultz, 2010).
Patient and Family Counseling Service is a social support resource for patients and their families at BCCA. It is offered automatically to those with responses that are indicative of anxiety or depression through PSSCAN screening. Patients can be referred by any healthcare worker or can choose to be refer themselves. The BCCC liaises between the patient and community resources and support services, when needed, and is available to meet with patients and their families face-to-face, over the phone, or via the Internet. The BCCC will also assist in the referral of patients to BCCA centre for oncology consultations when appropriate. The need for this BCCC to fulfil a navigator role was identified as a gap in care; a role was needed to provide patient information and supportive care for women from the beginning of their oncology consultation (Trussler, 2001).

Current or usual practice at BCCA is that all new patients seen at an initial visit are asked to complete the Psychological Screen for Cancer (PSSCAN) (Linden, Yi, Barroetavena, MacKenzie \& Doll, 2005; Linden, Vodermaier, MacKenzie, et.al., 2009). The PSSCAN is a 21-item questionnaire used to assess perceived social support, desired social support, health-related quality of-life, anxiety and depression. Psychological interventions for anxiety and depression, which may be identified as distress, can be utilized at the initial meeting to enhance quality-of-life, and help patients and families cope more effectively with a potential cancer diagnosis (Buxton, Lazenby, Daugherty, et.al., 2014). However, distress often remains unrecognized and, therefore, goes untreated (Carlson, Groff, Maciejewski \& Bultz, 2010). This screening instrument is to be completed by the patient prior to the oncology consultation, usually on the same day as the consultation. The PSSCAN results alert healthcare professionals to patients who may be experiencing higher levels of anxiety and depression. These patients and their family members are then referred to the patient and family counselling service at BCCA.

\section{METHODOLOGY}

A retrospective review of PSSCAN scores of new breast cancer patients seen for oncology consultation, between January 2010 and December 2012, who had not received care from a BCCC prior to the consultation visit (the control cohort) were compared to PSSCAN scores of those who had received care from a BCCC prior to the oncology consultation (the study cohort). Most of the referrals received by the BCCC were for patients who had abnormal SMP mammograms and were referred to the BHC for further investigations that may result in a breast cancer diagnosis. This study was restricted to these patients for both cohorts.

Inclusion criteria for the control cohort was as follows: a) patient presented with abnormal SMP mammogram; b) biopsy proven breast cancer diagnosis; c) completed the PSSCAN on initial BCCA oncology visit. The study cohort criteria were: a) patient presented with abnormal SMP mammogram; b) biopsy confirmed breast cancer diagnosis; c) received care from a BCCC prior to BCCA oncology consultation; d) completed the PSSCAN on initial BCCA oncology visit. Exclusion criteria for both cohorts were as follows: a) patient did not complete PSSCAN in full; b) patient presented with locally advanced or metastatic breast cancer; c) patient with previous breast cancer. 
PSSCAN Questionnaire: Reports indicate that utility of the PSSCAN as a screening tool, and possibly as a tool for tracking patient changes in emotional well-being, is sufficient because of its psychometric properties (Linden, Yi, Barroetavena, MacKenzie, \& Doll, 2005; Linden, Vodermaier, MacKenzie, et al., 2009). The PSSCAN is a 21-item psychological screening tool for use with cancer populations. The questionnaire is divided into three sections (i.e., A, B and C). The PSSCAN screening instrument has been utilized by BCCA as part of usual clinical practice for some time. However, in April 2012, BCCA evaluated the PSSCAN (2005) version and revised the PSSCAN version to the current PSSCAN-R version. The two versions of the PSSCAN and PSSCAN-R are identical in Part A with questions 1-5 remaining the same and question 6 being deleted. Part B had a complete change to the formatting of the questions, with Part $\mathrm{C}$ remaining entirely identical. Since these versions are somewhat different, we decided the responses from questions 1-5 in Part A and all the questions in Part C from PSSCAN would be included in this study in order to maintain similar data content to the revised content of the PSSCAN-R. This was important, especially since we are comparing efficacy of the PSSCAN responses. Subscales of the PSSCAN, for example subscale Part C, has been successfully used to measure anxiety and depression with lung and breast cancer patients (Carlson, Groff, Maciejewski, \& Bultz, 2010). Unfortunately, no other studies utilizing PSSCAN-R were found to validate that the revised version has been successful.

In light of the utility of the PSSCAN, the null hypothesis is: New patients completing the PSSCAN questionnaire and receiving supportive care from the BCCC prior to the oncology consultation visit demonstrate similar levels of anxiety and depression as those patients who only completed the PSSCAN questionnaire.

In addition to the PSSCAN scores for each patient, demographic data were collected as potential variables affecting PSSCAN scores. These data included: age at diagnosis; living arrangements; marital status, social support; financial concerns, care of dependents, language barrier (interpreter requirement) and past psychological history.

\section{Data analysis}

For the purposes of analysis, a numerical value was assigned to each of the questions. Descriptive statistics were used to identify differences between the two groups of participants. This analysis provides a comparison of responses to PSSCAN scores between the two groups in order to identify any subjective differences of patients' experiences as they initially entered the BHC of BCCA. All analyses were conducted using SAS, version 9.3 (Hatcher, 2003).

\section{RESULTS}

A total of 91 patient PSSCAN records were reviewed for this study, with 54 belonging to the treatment study group and 37 in the control group. Demographic and social support data are presented in Table 1. Women assigned to the control group were significantly more likely to report that they lived alone. Women assigned to the treatment group were significantly more likely to report that they had dependents who counted on them for care and assistance. These were the only two demographic variables that differed significantly between the two groups. There were no differences between the groups on language difficulties $(\mathrm{c} 2(1)=1.58, \mathrm{p}=.20)$, on having past psychiatric diagnoses $(\mathrm{c} 2(1)=.0057, \mathrm{p}=.93)$, or on whether they were exposed to past financial concerns (PFC) or not $(\mathrm{c} 2(1)=1.45$, $\mathrm{p}=.22$ ).

Mean scores on the PSSCAN items for anxiety and depression can be found in Table 2. There was only one item for which either group, in this case the treatment group, had a significantly higher mean score; this was for the item "During the past week I have lost interest in things I usually cared for or enjoyed."

Utilizing the data from the two PSSCAN subscales for which we had similar data, social support and anxiety/depression, independent samples t-tests were run to determine differences in mean scores between the control and study groups. For the social support subscale, women in the control group scored significantly higher $(\mathrm{M}=3.36, \mathrm{SD}=0.76)$ compared to the study group $(\mathrm{M}=2.96, \mathrm{SD}=0.60, \mathrm{t}(59)=2.62, \mathrm{p}=.01)$. For the anxiety/depression subscale, there were no differences between the scores for the control group $(\mathrm{M}=11.91, \mathrm{SD}=3.70)$ compared to the study group $(\mathrm{M}=12.77, \mathrm{SD}=5.89$, $\mathrm{t}(88)=\mathrm{ns})$.

\section{DISCUSSION}

This study explored the impact on distress of a Breast Cancer Care Co-ordinator (BCCC) program that offered support for women in the critical transition between being identified of having a suspicious tumour and an actual diagnosis. The unique healthcare partnership and collaboration between the hospital setting and the BCCA centre created an opportunity to provide patient information and supportive care for women with suspicion of breast cancer to an actual diagnosis. Utilizing BCCCs who are oncology nurses in this program permitted continuous cancer care and a navigational support for women from the beginning of the cancer care trajectory.

The BCCC is seen as an important link to the interdisciplinary team and allows for liaison with the community resources for the patient. Furthermore, the BCCC is able to introduce the Patient and Family Counseling, a social support resource at BCCA, as needed. Development of a clinical pathway with consistent nursing care during the cancer trajectory is expected to be helpful in reducing distress and building trust and rapport. This strategy of continuous cancer care may also result in better uptake of cancer diagnosis and treatment-related information, and perhaps a better understanding of the follow-up care post-treatment (Zabora, Brintzenhofeszoc, Curbow, Hooker, \& Piantadosi, 2001). These aspects will need to be evaluated using relevant variables for a more in-depth study of the impact of the BCCC role in the future.

Although results in this study do not show a significant difference regarding distress between the treatment and cohort groups at the time of the initial oncology consultation following a positive diagnosis, timing of the data capture may be the reason. Women completed the PSSCAN before their initial consultation with the oncologist when they would be 


\begin{tabular}{|l|l|c|c|}
\hline $\begin{array}{l}\text { Table 1: Treatment versus control group (demographics and social } \\
\text { support) }\end{array}$ & $\begin{array}{c}\text { Treatment } \\
(\mathbf{N}=54)\end{array}$ & $\begin{array}{c}\text { Control } \\
(\mathbf{N}=37)\end{array}$ & c2 \\
\hline Variable & N (\%) & N (\%) & \\
\hline & Mean (SD) & $\begin{array}{c}\text { Mean } \\
(\text { SD })\end{array}$ & t \\
\hline Age & $\begin{array}{c}62.05 \\
(10.1)\end{array}$ & $\begin{array}{c}65.9 \\
(8.42)\end{array}$ & 1.93 \\
\hline Time between assessment/TX & $\begin{array}{c}87.84 \\
(28.89)\end{array}$ & $\begin{array}{c}83.16 \\
(29.24)\end{array}$ & 0.75 \\
\hline
\end{tabular}

Did you need an interpreter?

\begin{tabular}{|l|c|c|c|}
\hline Yes & $5(100)$ & $0(0)$ & \\
\hline No & $48(56)$ & $37(44)$ & $3.69^{*}$ \\
\hline
\end{tabular}

Dependents counting on you for care?

\begin{tabular}{|l|l|l|l|}
\hline Yes & $12(86)$ & $2(14)$ & \\
\hline No & $41(55)$ & $34(45)$ & $4.72^{* *}$ \\
\hline Do you live alone? & $6(33)$ & $12(67)$ & \\
\hline Yes & $47(65)$ & $25(35)$ & $6.07^{* * *}$ \\
\hline No &
\end{tabular}

When you need help can you count on anyone to help with daily tasks such as shopping, cooking, giving you a ride?

\begin{tabular}{|c|c|c|c|}
\hline Yes & $44(56)$ & $35(44)$ & \\
\hline No & $7(78)$ & $2(22)$ & 1.61 \\
\hline \multicolumn{5}{|l|}{ Do you have regular contact with friends or relatives? } \\
\hline Yes & $53(60)$ & $36(40)$ & \\
\hline No & $1(50)$ & $1(50)$ & 0.07 \\
\hline
\end{tabular}

Have you lost your life partner within the last few years?

\begin{tabular}{|c|l|l|l|}
\hline Yes & $4(44)$ & $5(55)$ & \\
\hline No & $47(61)$ & $30(39)$ & 0.91 \\
\hline
\end{tabular}

Can you count on anyone to provide you with emotional support?

\begin{tabular}{|l|c|c|c|}
\hline Yes & $50(58)$ & $36(42)$ & \\
\hline No & $3(75)$ & $1(25)$ & 0.44 \\
\hline
\end{tabular}

Note. ${ }^{*} \mathrm{p}<.10 ;{ }^{* *} \mathrm{p}<.05 ;{ }^{* * *} \mathrm{p}<.01$.
Table 2: Mean responses to recent and past experiences of anxiety and depression

\begin{tabular}{|c|c|c|c|}
\hline Variable & $\begin{array}{l}\text { Treatment } \\
(N=54)\end{array}$ & $\begin{array}{l}\text { Control } \\
(\mathrm{N}=37)\end{array}$ & $t$ \\
\hline Mean (SD) & Mean (SD) & & \\
\hline $\begin{array}{l}\text { During the past week, I } \\
\text { have felt my heart race and } \\
\text { I tremble }\end{array}$ & $1.17(.69)$ & $1.16(.55)$ & 0.03 \\
\hline $\begin{array}{l}\text { During the past week, I } \\
\text { have felt that I cannot } \\
\text { control anything }\end{array}$ & $1.33(.93)$ & $1.14(.42)$ & 1.21 \\
\hline $\begin{array}{l}\text { During the past week, I } \\
\text { have lost interest in things I } \\
\text { usually cared for or enjoyed }\end{array}$ & $1.29(.71)$ & $1.03(.37)$ & $2.10^{*}$ \\
\hline $\begin{array}{l}\text { During the past week, I } \\
\text { have felt nervous and shaky } \\
\text { inside }\end{array}$ & $1.35(.82)$ & $1.39(.86)$ & 0.15 \\
\hline $\begin{array}{l}\text { During the past week, I } \\
\text { have felt tense and cannot } \\
\text { relax }\end{array}$ & $1.41(.90)$ & $1.37(.75)$ & 0.16 \\
\hline $\begin{array}{l}\text { During the past week, my } \\
\text { thoughts are repetitive and } \\
\text { full of scary things }\end{array}$ & $1.22(.90)$ & $1.16(.60)$ & 0.35 \\
\hline $\begin{array}{l}\text { During the past week, I } \\
\text { have felt restless and find it } \\
\text { difficult to sit still }\end{array}$ & $1.29(.81)$ & $1.21(.47)$ & 0.54 \\
\hline $\begin{array}{l}\text { I have recently thought } \\
\text { about taking my life }\end{array}$ & $0.92(.38)$ & $0.95(.32)$ & 0.26 \\
\hline $\begin{array}{l}\text { In the past year, I have had } \\
2 \text { or more weeks during } \\
\text { which I felt sad, blue or } \\
\text { depressed }\end{array}$ & $1.46(1.09)$ & $1.35(.89)$ & 0.51 \\
\hline $\begin{array}{l}\text { I have had two years or } \\
\text { more of my life when I } \\
\text { felt depressed or sad most } \\
\text { days even if I felt okay } \\
\text { sometimes }\end{array}$ & $1.31(.94)$ & $1.16(.72)$ & 0.83 \\
\hline
\end{tabular}

learning more about their diagnosis and treatment options. Undoubtedly anxiety would be high at this point. In the future, collecting the distress data at another point in time would perhaps be more appropriate.

A systematic review of patient navigation in breast cancer, reveals that navigation across many aspects of the breast cancer disease trajectory and the BCCC role improves many positive aspects of breast cancer care (Case, 2011; Robinson-White, Conroy, Slavish, \&Rozenzweig, 2010). The role of a breast cancer nurse navigator is reported as a key role that helped 
allay anxiety and fear for women as they received an incredible amount of information upon entry into the organizational structure of the cancer treatment (Trevillion, Singh-Carlson, Wong, \& Sherriff, 2015). Additional work is needed to understand the role and its patient outcomes surrounding the diagnostic period.

Psychological support at the new appointment is important. However, a study on the effectiveness of the PSSCAN-R screening instrument at both an initial and post oncology consultation would be important to identify any differences in anxiety levels. Multiple administrations of the PSSCAN-R (i.e., at the initial diagnostic visit and subsequent follow-up visits, as necessary) would help to evaluate an effect change with the baseline and follow-up data. Carlson, Groff, Maciejewski and Bultz (2010) support this study by reporting that breast cancer patients showed lower distress at follow-up than those at initial screening. Other reports indicate that screening for and management of distress generally leads to better patient outcomes and an awareness of screening will prompt cancer patients to speak to oncologists about their anxiety and depression (Buxton, Lzenby, Daugherty, et.al., 2014). By openly discussing potential emotional distress, patients will have higher rates of psychosocial self-care and feel more satisfied with their cancer treatments; thereby living a better quality of life. Again, these aspects need to be considered in light of the distress surrounding the diagnostic period.

This study focused on determining whether patients receiving navigational supportive care and information from a BCCC prior to initial oncology consultation at a BCCA centre demonstrated any different levels of anxiety and depression from those not receiving such support at the same BCCA centre. National Coalition of Oncology Nurse Navigators (2017) supports the role of the breast cancer care navigator who can help alleviate anxiety experienced by an abnormal mammogram. The nurse, through a demonstration of interpersonal communication skills, can advocate for patients by engaging with them through written, spoken and active listening skills that promote patient-centred care. Early and effective assessment of readiness to learn, learning styles, and distress levels are important during the diagnostic period in order to offer information and support effectively and in a culturally sensitive manner.

\section{Limitations}

Some limitations of the current study should be noted. First, the sample size is small and a larger group of women receiving the nurse navigation assistance might reveal differences not detected in the current study. Secondly, distress is only one outcome in relation to the role of the BCCC, Other variables need to be included in future studies. Thirdly, only utilizing data from subscales A and C of PSSCAN and PSSCAN-R and deleting the data for question 6 from Part A of the PSSCAN instrument created complexities in data elicitation of the retrospective chart review. The change to the PSSCAN format during the study was an issue. The shortened version of the PSSCAN-R has not been subjected to reliability and validity testing yet. While the psychometric values of the original PSSCAN have been established, we cannot conclude for certain that the reduced number of items in the PSSCAN-R used in this study retained the original reliability and validity. Finally, the manual retrospective review of the PSSCAN scores between January 2010 and December 2012 was challenging in that identifying the control cohort using the inclusion/exclusion criteria presented difficulties, resulting in the smaller sample size.

\section{RECOMMENDATIONS AND IMPLICATIONS FOR NURSING}

This study provides an opportunity to reveal the important role of a BCCC in the cancer setting for women as they are first introduced to a cancer clinical setting. Connecting with the patient at the initial visit and helping to introduce them to the procedures and process is helpful for the patient. However, exactly how this help is realized and the relevant variables might need to be explored using qualitative approaches. This would highlight the impact of the BCCC role in the cancer setting from a wider perspective other than distress.

Utility of the PSSCAN as the questionnaire to measure anxiety and depression for patients entering the BCCA organization is an adequate protocol. However, future recommendations would be to conduct an evaluative study to measure the change in the PSSCAN survey post oncology consult, as well as establishing the reliability and validity of the reduced number of items reflected in the PSSCAN-R.

Women have identified that a phone call prior to the initial oncology consultation, as well as in follow-up after that initial clinical visit, is beneficial (Trevillion, Singh-Carlson, Wong, \& Sherriff, 2015). This allowed for a connection with a healthcare provider at the cancer centre clinic, thereby reducing some anxiety about the unknown. As part of the collaborative interdisciplinary team, the BCCC's ability to be attentive to the patient's need for information in light of the diverse populations is critical. The BCCC strategy of supporting patients at their initial oncology consultation, can be the basis for establishing a trusting and caring nurse-patient relationship that exemplifies a patient-centred approach. 


\section{REFERENCES}

British Columbia Cancer Agency (2017). Intelligent patient guide to breast cancer, 6th edition. Retrieved from http://www.bccancer. bc.ca/health-info/types-of-cancer/breast-cancer/information-kit

Buxton, D., Lazenby, M., Daugherty, A., Kennedy, V., Wagner, L., Fann, J.R., \& Pirl, W. (2014). Distress screening for oncology patients. Oncology Issues Journal, January-February 2014, 49-52. Retrieved from www.accc-cancer.org

Campbell, C., Craig, J., Eggert, J., \& Bailey-Dorton, C. (2010). Implementing and measuring the impact of patient navigation at a Comprehensive Community Cancer Center. Oncology Nursing Forum, 37(1), 61-68.

Carlson, L.E., Groff, S.L., Maciejewski, O., \& Bultz, B.D. (2010). Screening for distress in lung and breast cancer outpatients: A randomized controlled trial. Journal of Clinical Oncology,25(933), 4884-4891.

Case, M.A.B. (2011). Oncology nurse navigator: Ensuring safe passage. Clinical Journal of Oncology Nursing, 15(1), 33-40.

Drageset, S., Lindstrom, T.C., \& Underlid, K. (2009). Coping with breast cancer: Between diagnosis and surgery. Journal of Advanced Nursing, 66(1), 149-158.

Fillion, L., Cook, S., Veillette, A., Aubin, A., De Serres, M., Fitch, M., \& Doll, R. (2012). Professional navigation framework: Elaboration and validation in a Canadian context. Oncology Nursing Forum, 39(1), E58-69.

Hatcher, L. (2003). Step-by-step basic statistics using SAS. Cary, N.C.: SAS Institute Inc.

Hewitt M, Greenfield S, Stovall E (Eds.). (2005). From cancer patient to cancer survivor: Lost in transition. Washington, DC: National Academies Press.

Hryniuk, W., Simpson, R., McGowan, A., \& Carter, P. (2014). Patient perceptions of a comprehensive cancer navigation service. Current Oncology, 21, 69-76.

Liebert, B., Parle, M., White, K., \& Rodger, K. (2001). Establishing an evidence base for the specialist breast nurse: A model for Australian breast cancer care. Australian Health Review, 24(1), 192-200.

Lim, C.C., Devi, K., \& Ang, E. (2011). Anxiety in women with breast cancer undergoing treatment: A systemic review. International Journal of Evidence-Based Healthcare, 9, 215-235.
Linden, W., Yi, D., Barroetavena, M.C., MacKenzie, R., \& Doll, R. (2005). Development and validation of a psychosocial screening instrument for cancer. Health and Quality of Life Outcomes, 3, 54.

Linden, W., Vodermaier, A.A., MacKenzie, R., Barroetavena, M.C., Dahyum, Y., \& Doll, R. (2009). The psychosocial screen for cancer (PSSCAN): Further validation and normative data. Health and Quality of Life Outcomes, 7(16), 1-8.

National Cancer Institute. (2017). Follow-up care after cancer treatment. Retrieved from http://www.cancer.gov/about-cancer/coping/ survivorship/follow-up-care/follow-up-fact-sheet

National Coalition of Oncology Nursing Navigators. (2017). Who is the nurse navigator? Retrieved from http://www.nconn.org/

Pederson, A.E., Jo-Ann Sawatzky, J.A., Hack, T.F. (2010). The sequelae of anxiety in breast cancer: A human response to illness model. Oncology Nursing Forum, 37(4), 469-475.

Pedersen, A.E., \& Hack, T.F. (2011). The British Columbia Patient Navigation Model: A critical analysis. Oncology Nursing Forum, 38(4), 200-206.

Robinson-White, S., Conroy, B., Slavish, K.H., \& Rosenzweig, M. (2010). Patient navigation in breast cancer. Cancer Nursing, 33(2), 127-140.

Singh-Carlson, S., \& Gotz, C. (2014). Breast cancer survivorshipoptimizing follow-up care: Patients' perspectives of their practical needs. Journal of Women's Health, Issues \& Care, 3(1). Retrieved from http://www.scitechnol.com/breast-cancer-survivorshipoptimizing-followup-care-patients-perspectives-practical-needsqXS0.pdf?article_id=1750

Trevillion, K., Singh-Carlson, S., Wong, F., \& Sherriff, C. (2015). An evaluation of the nurse navigator services for the breast cancer support program. Canadian Oncology Nursing Journal, 25(4), 409-414.

Trussler, T. (2001). Uncovering the gaps: An inquiry of breast care in British Columbia. Canadian Breast Cancer Foundation BC/Yukon Chapter.

Winstead, E.R. (2012). As patient navigation expands and evolves, nurses assume many roles. National Cancer Institute, 9(15).

Zabora, J., Brintzenhofeszoc, K.B., Curbow, C., Hooker, C., \& Piantadosi, S. (2001). The prevalence of psychological distress by cancer site. Psycho-Oncology 10, 19-28. 